\title{
Sixth National Scientific Workshop of the Muscular Dystrophy Association of Canada
}

\author{
ABSTRACTS
}

\section{The Synthesis of Z-Line Proteins in Skeletal Muscle of Nor- mal and Dystrophic Mice.}

B.G.ATKINSON, R.R.SHIVERS and M.POLLOCK and J. RUTLEDGE. Cell Science Laboratories, Dept. of Zoology, University of Western Ontario, London, Ontario.

Electron microscopic ultrastructural and freeze fracture examination of skeletal muscle from dystrophic and normal mice $(\mathrm{ReJ} / 129)$ disclose that the plasma membrane of dystrophic muscle exhibits a marked reduction in intramembrane particles (PF face particles) and numerous enlarged multiple exocytotic profiles, and that the myofibrils exhibit a high deficiency in and/or a marked streaming of the myofibrillar Z-line structure. These ultrastructural observations prompted biochemical studies aimed at furthering our understanding of the protein constituents found in the skeletal muscle Z-lines of normal mice and found and/or deficient in the skeletal muscle Z-lines of dystrophic mice. By sensitive fluorographic analysis of one- and two-dimensional polyacrylamide gel-separated muscle proteins and immunochemical techniques we have compared and assessed the diversity, and, in some cases, the quantity and rate of synthesis of specific myofibrillar and sarcoplasmic proteins. Our results demonstrate an early (newborn dystrophic mice) and progressive decrease in the synthesis of $\alpha$-actinin and possibly other Z-line proteins (desmin and vimentin), whereas a decrease in the quantity and synthesis of other myofibrillar proteins is not evident in skeletal muscle of dystrophic animals until at least 1 month after birth. We contend that the early and progressive depressed synthesis of Z-line proteins in dystrophic muscle results in Z-line 'streaming' and myofibrillar disassembly and muscle dysfunction. Supported by a grant from MDAC.

\section{Monocyte IgG-Fc Receptor Concentration and Affinity in Myotonic Dystrophy.}

DIPONKAR BANERJEE, JEFFREY MCCLINTOCK, MEREDITH M. SILVER and ARTHUR J. HUDSON. Departments of Pathology and Clinical Neurological Sciences, Faculty of Medicine, University of Western Ontario, London, Canada.

In patients with myotonic dystrophy (MyD), serum IgG levels are subnormal due to hypercatabolism of $\mathrm{IgG}$, unrelated to structural defects in the IgG molecule. The normal concentration-fractional catabolic rate relationship of IgG does not appear to hold true in $\mathrm{MyD}$. IgG half-life in MyD patient is approximately half of that in normal individuals, and the IgG fractional catabolic rate is approximately twice that in normals. We have postulated that a species of monocyte IgG Fc receptors are "protector" molecules which bind to IgG and prevent its uptake into its catabolic compartment, and that in MyD patients this "protector" molecule is defective due to low receptor concentrations or low affinity. Purified peripheral blood monocytes from $9 \mathrm{MyD}$ patients and 13 normal subjects were analyzed for receptor concentration and affinity by Scatchard analysis of ${ }^{125} \mathrm{I}-\mathrm{IgG}$ binding in vitro. We found that $\mathrm{MyD}$ monocytes express more receptors per cell $(45,200)$ than normal monocytes $(27,600)$ but bind with lower affinity $\left(\mathrm{Ka}=1.5 \times 10^{8} \mathrm{M}^{-1}\right.$ for $\mathrm{MyD}$ monocyte $\mathrm{Fc}$ receptors and $2.4 \times 10^{8} \mathrm{M}^{-1}$ for normal monocytes). These findings support the hypothesis that hypercatabolism of IgG in MyD may be due to low affinity of the "protector" Fc receptors on monocytes. Supported by a grant from MDAC (M335A1).

\section{Properties of Motor Units in Dystrophic Mouse EDL.}

\author{
D.S. BATESON and DJ. PARRY, Univ. Ottawa, Ottawa, Ont.
}

The extensor digitorum longus muscle (EDL) of mouse consist almost entirely of type II fibres (fast twitch). The motor unit composition of EDL in normal and dy $2 \mathrm{~J}$ mice has been examined. 64 units in 15 normal mice and 60 units in 15 dystrophic mice were studied. Motor units were isolated by ventral root splitting of $L 3 \&$ L 4 roots. Motor unit size was expressed as unit tetanic tension as a percentage of whole muscle tetanic tension. Both normal and dy $2 \mathrm{~J}$ mice showed little variation; $1.0 \%$ to $11.7 \%$ (mean value \pm S.D. $=4.6 \pm 2.5 \%$ ); $1.0 \%$ to $18.4 \%$ (mean value \pm S.D. $=6.7 \pm$ $4.1 \%)$ respectively. This suggests that the C57 EDL contains about 22 motor units while dy $2 \mathrm{~J}$ EDL is composed of about 15 . Both normal and dy $2 \mathrm{~J}$ mice exhibit a unimodal distribution of unit times to peak tension (CT). For normal units the mean value was $8.7 \pm 1.2 \mathrm{msec}$. For dystrophic units the mean CT was $11.9 \pm 2.7 \mathrm{msec}$. Mean axonal conduction velocity for normal mice was $70.1 \mathrm{~m} / \mathrm{sec}$. and $46.3 \mathrm{~m} / \mathrm{sec}$. for dystrophic mice. No simple relationships were observed between conduction velocity and either twitch or tetanic tension or CT. Supported by MDAC.

\section{Histamine and Mast Cell Abnormalities in Avian and Murine Muscular Dystrophy.}

A. DEAN BEFUS and LAURIE NIELSEN. McMaster University, Hamilton, Ontario.

In avian and murine muscular dystrophy there are abnormalities in thymic development and in mast cell and histamine levels in various tissues. Since the thymus is a rich source of mast cells and the histamine they contain, we conducted an ontogenetic analysis of thymic mast cells and histamine levels in avian and murine muscular dystrophy. In the avian thymus, two abnormalities were detected: firstly, from hatching to 3 wk of age the histamine and mast cell concentration was significantly reater in the dystrophic than in the normal bird; and secondly, in dystrophic birds $>3$ wk, the histamine and mast cell concentration was significantly lower than normal. By contrast, in murine muscular dystrophy $\left(\mathrm{dy}^{2 J}\right)$, rather than a histamine deficiency in older animals as in the bird, there was a significant increase above normal in thymic histamine concentration. Histamine and mast cell abnormalities were detected in other tissues as well, perhaps most notably there was a significant increase in affected muscle in both mice and chickens. The abnormalities in the thymus and affected muscle may be important in the genesis of the dystrophic process and in the degenerative events in affected muscle. (Supported by a grant from MDAC.)

\section{Comprehensive Neuromuscular Assessment in Myotonic Dystrophy.}

A.Y. BELANGER* and A.J. McCOMAS. McMaster University Medical Centre, Hamilton, Ontario.

Although myotonic dystrophy is now the most common type of dystrophy, a systematic study of neuromuscular function has not previously been reported. We have examined the electrical and contractile behaviour of ankle dorsi-flexor (DF) and plantar-flexor (PF) muscles in 25 patients and in a similar number of closely-matched control subjects. In keeping with expectation, there was a reduction in excitable muscle mass in DF and PF muscles as shown by diminished M-waves, isometric twitches and voluntary torques. There was a tendency for relatively greater involve- 
ment of DF than PF muscles in severely affected patients; in both muscle groups, however, surviving fibres had normal twitch-potentiation and fatigue properties. In comparison with control subjects the PF muscles were abnormal in having faster twitches and poor motor unit activation. This type of comprehensive testing should enable the timecourse of the dystrophic process to be determined, together with the effectiveness of experimental therapy. Supported by a grant from MDAC.

\section{Muscle Function Studies in Human Plantar-Flexor and Dorsi-Flexor Muscles.}

A.Y. BELANGER and J. QUINLAN*. McMaster University Medical Centre, $\mathrm{H}$ amilton, Ontario.

Methods have been developed for studying contractile function in human leg muscles. By judicious placement of stimulating electrodes and by applying initial stretch to the muscle group under study, it has been possible to isolate the mechanical responses of the plantar-flexor (PF) and dorsi-flexor (DF) muscles of the ankle. In healthy young adults of both sexes it has been found that the PF muscles, of which the triceps surae is the most powerful, have relatively slow twitches, weak post-activation potentiation, and high resistance to fatigue; however, full motor unit activation is not easily achieved. In comparison the tibialis anterior, the DF muscle studied in most detail, has a faster twitch, marked post-activation potentiation, and is very susceptible to fatigue; complete voluntary activation of the muscle is easily obtained. The differences between the two muscle groups are partly dependent on fibre typing and therefore make the muscles very suitable for comparison of involvement in various neuromuscular disorders.

\section{Chick-Quail Chimaeras as Models for Ex Ovo Muscle Dif- ferentiation.}

B. BERESFORD, J.BUTLER, E. COSMOS and J. BIENENSTOCK. Depts. Pathology and Neurosciences, McMaster Univ., H.S.C., Hamilton, Ontario.

The chick-quail chimaera provides embryologists with the ideal marker to study the origin, migration, and destination of specific tissues during early stages of embryogenesis. However, such chimaeras have not been maintained and analyzed ex ovo to determine whether the foreign tissue survives to later stages, unaffected by the host immune system. Ex ovo chimaeras would provide a useful model to study the later differentiation of tissues such as muscle, to determine whether the pheno-typic characteristics expressed by the foreign tissue are intrinsic to it or are modified by the host environment. As a preliminary study, we wish to report the analysis of one such chimaera maintained to 3 months ex ovo. Brachial somites 16 to 21 of a quail embryo were transplanted isotopically to a chick embryo at 2 days in ovo. Analysis of several brachial muscles of the adult chimaera indicated: 1) successful survival of the grafted tissues; 2) only brachial muscles were of quail genotype, confirming in ovo studies; and 3) the distinctive quail muscle fiber type enzymatic profiles were expressed despite their innervation by chick peripheral motoneurons. If further experiments confirm the suitability of the ex ovo chimaera, similar chimaeras can be used to study the ex ovo differentiation of muscle of dystrophic genotype in the host environment of the normal, and vice versa. Supported by grants from the MDAC and MDA.

\section{Experimental Approaches to the Study of Nerve Influences on Fast and Slow Muscle During Early Embryogenesis in the Chicken.}

J.W. BLOOM and E. COSMOS. McMaster University Health Sciences Centre, Hamilton, Ont.

The musculature of the brachial region of the chick embryo differentiates anatomically and histochemically in the complete absence of innervation, but fails to grow and, by day $10-11$, begins to degenerate (1). Therefore some neural influence, activity dependent and/or trophic, is necessary for muscle growth during the first half of development. We are using three experimental manipulations to modify nerve muscle interactions during this period. Neural tube removal eliminates trophic and activity effects. Pharmacological neuromuscular block ade (e.g., by curare) removes activity ef- fects without altering trophic effects. The degree of inactivity induced is assessed by observation of the motility of embryos within windowed eggs. Electrical stimuation via the nervous system augments activity without altering trophic effects while direct electrical stimulation of aneural muscle supplies activity in the absence of trophic substances. Anterior and posterior latissimus dorsi muscles (slow tonic and fast twitch respectively) subjected to these manipulations are examined by quantitative histological and electron microscopic methods to assess their effects on early muscle growth.

(1) Butler, J., J. Brierley, E. Cosmos (1980). Am. Zool. 20:805. Supported by grants from MDAC and MDA.

\section{Pertubations in the Protein Synthesis Activity of Polysomes from Dystrophic Hamsters.}

LÉA BRAKIER-GINGRAS and CHRISTINE JOLICOEUR, Département de Biochimie, Université de Montréal.

Polysomes have been isolated from dystrophic hamsters at various stages of the disease (30,60,120 and 200 days) and from age-matched healthy controls. Polysomes have been extracted from the heart, the skeletal muscle and the liver and their activity has been assayed in a cellfree system, using a wheat germ extract or a reticulocyte lysate as a source of soluble factors. The results show that the activity of polysomes from the heart and the skeletal muscle, but not from the liver, of dystrophic hamsters is decreased with respect to controls. The decrease is age-dependent: with skeletal muscle polysomes, it is observed at 30 days, disappears at 60 days, but reappears in older animals; with heart polysomes, it is observed at 60 days but not in younger or older animals. The decrease is also observed when endogenous messengers are replaced by poly (U), suggesting that the defect may be related to the ribosomes. Supported by a grant from MDAC.

\section{Enhanced Axonal Ensheathment in Regenerated Spinal Roots of Dystrophic Mice}

By G.M. BRAY, T. CARLSTEDT, S. DAVID, W. WILCOX and A. J. AGUAYO. Neurosciences Unit, The Montreal General Hospital and McGill University.

Schwann cells (SC) in the lumbosacral spinal roots of dystrophic mice fail to ensheath many axons during primary development but when dystrophic roots are grafted into peripheral nerves, the regenerated axons are normally ensheathed. To determine whether the dystrophic spinal root abnormality is due to its location or its time of onset, we have examined dorsal spinal roots in dystrophic mice after crush injury. Although the regenerated roots showed decreased total numbers of axons and increased amounts of collagen, all axons were ensheathed as myelinated and unmyelinated fibers; the characteristic groups of unensheathed axons did not redevelop. These results suggest that the location of the dystrophic abnormality is not the exclusive mechanism responsible for its development. Supported by a grant from MDAC.

\section{Contractile Properties of Dystrophic Skeletal Muscle.}

B.H. BRESSLER, W.K. OVALLE, L.G. JASCH, C.E. SLONECKER. Department of Anatomy, University of British Columbia, Vancouver, B.C.

Previous studies from our laboratory have revealed that dystrophic slow-twitch SOL, of the C57BL/6J dy $2 \mathrm{~d} / \mathrm{dy}^{2 \mathrm{~J}}$ strain of dystrophic mice generate less isometric twitch and tetanus tension than their normal counterparts. The dystrophic fast-twitch EDL produced less isometric twitch and tetanus tension at 4 and 8 weeks of age whereas by 32 weeks of age there was no difference seen between normal and dystrophic muscles. By 8 weeks, dystrophic EDL exhibited a prolonged time-to-peak twitch tension (TTP) and half-relaxation time $(1 / 2 \mathrm{RT})$ which continued to 32 weeks. At 8 and 12 weeks, TTP and $1 / 2$ RT of dystrophic SOL were prolonged. However, by 32 weeks there was no difference in the TTP between normal and dystrophic SOL and a significant speeding up was recorded in the $1 / 2$ RT. Preliminary experiments at 52 weeks of age produced similar results in terms $1 / 2 \mathrm{RT}$ of the dystrophic SOL to those seen at 32 weeks. Studies on denervated muscle from normal mice (Webster and Bressler, unpublished) have demonstrated that changes in 
contractile function of denervated EDL are remarkably similar to those changes seen in dystrophic EDL. The possibility that dystrophy may be the result of a lack of a proper neurotrophic influence will be discussed. Supported by MDAC.

\section{Response of Brachial Muscles to Heterotopic Innervation During Embryogenesis.}

J. BUTLER, E. COSMOS, and J. BRIERLEY. McMaster University, Health Sciences Center, Hamilton, Ontario.

Since avian HMD is fiber type specific, we are investigating factors in volved in the incipient expression of fiber types in both normal and dystrophic embryos. It is well documented that ex ovo nerves can alter fiber-type characteristics. To determine if in ovo nerves function similarly, heterotopic innervation experiments were performed. The brachial segment of the neural tube was extirpated at $48-52 \mathrm{hr}$ and replaced by the thoracic segment of a donor embryo ( $50-53 \mathrm{hr}$ ), or, as controls, a brachial segment. Analyses indicate that grafts survive, brachial plexii form and individual muscles expresss appropriate fiber types. Furthermore, from day- 4 to day11 , both control and experimental muscles are innervated and equivalent in size. However, from day-12 to day- 19 in ovo, within the heterotopic experiments, the muscle-nerve union uncouples, muscles progressively degenerate and are replaced by lipid. Thus, inappropriate nerves do support normal muscle growth during the first half of embryogenesis, but, do not alter fiber types or establish permanent neuromuscular junctions during the second half of embryogenesis. Suppored by grants from MDAC and MDA.

\section{Experimental Micropuncture Injury of Skeletal Muscles.}

STIRLING CARPENTER, GEORGE KARPATI, Department of Neurology-Neurosurgery, McGill University, Montreal Neurological Institute, Montreal, Quebec.

The exposed gastrocnemius muscle of anesthetized rats is subjected to multiple punctures by a tungsten wire $10 \mu \mathrm{m}$ in diameter. Muscles are sampled at times ranging from less than 5 minutes to 4 days, and are studied in cryostat, semithin resin and ultrathin sections. Right after puncture, the superficial part of the punctured tract is bordered by a collar of intense hypercontraction, which is not present deeper in the fiber. Dilated membrane-bound spaces are found for some distance around the puncture in the fiber. By 6 hours there is extensive segmental necrosis. Around this time a membrane is rapidly generated which divides necrotic from surviving segments. Vacuolation then rapidly disappears in the surviving segments. By 72 hours regenerative myoblasts line the old basal lamina tube. At 96 hours continuity of the muscle fiber has been re-established across the area where necrosis took place. Supported by a grant from MDAC.

\section{Sexual Dimorphism in the Cellular Immune Response of Dystrophic Mice.}

S. COLBY-GERMINARIO, W. BINDON and B.G. LIVETT, The Montreal General Hospital and McGill University, Montreal Quebec.

129ReJ $(d y / d y)$ and C57B 1/6J $\left(d y^{2 \mathrm{~J}} / d y^{2 J}\right)$ mice exhibit a reduced cellular immune response as evidenced by a reduced capacity to reject skin grafts (Colby-Germinario et al, 1980). This deficiency is observed in 3-6 week old mice but is not detectable in mice over 12 weeks of age.

To further examine this developmental defect we have used an in-vitro measure of the cellular immune response (phytohemagglutinin induced lymphoblastic transformation (LBT) of splenic lymphocytes) in 6 week old C57B 1/6J $( \pm / \pm)$ and $\left(d y^{2} / d y^{2 J}\right)$ mice. No significant difference in the LBT response was found. However, when the male and female responses were analyzed separately, a marked sexual dimorphism was apparent. The LBT response (stimulation index) of dystrophic females $(11.86 \pm 1.41, n=4)$ was significantly less $(p<0.001)$ than that of normal females $(26.2 \pm 4.7$, $\mathrm{n}=3)$. In direct contrast, the response of dystrophic males $(27.4 \pm 14.7$, $n=5)$ appeared higher ( $p>0.05$ N.S.) than normal males $(14.4 \pm 6.3$, $\mathrm{n}=5$ ) and far more variable. Although a full developmental study will be required, these preliminary in-vitro results are consistent with the in-vivo evidence for a developmental defect in the cellular immune response in young dystrophic mice.
Colby-Germinario, S., Martial, E. and Livett, B.G. (1980). Can. J. Neurol. Sci. 7, 124. Supported by a grant from MDAC.

The Interactions of Skeletal Muscle, Smooth Muscle and Non-Muscle Tropo-Myosins with F-Actin and Skeletal Muscle Troponin and its Components.

G.P. CÔTÉ, A.S. MAK, J.R. PEARLSTONE, C. SANDERS and L.B. SMILLIE. MRC Group in Protein Structure \& Function, Dept. of Biochemistry, University of Alberta, Edmonton.

Skeletal and smooth muscle tropomyosins (TM) bind similarly to F-actin as a function of $\left[\mathrm{Mg}^{2+}\right]$ and aggregate extensively in a head-to-tail manner. Platelet TM requires higher $\left[\mathrm{Mg}^{2+}\right](\sim 8-10 \mathrm{mM})$ and aggregates poorly. These differences are attributable to the shorter length of the platelet TM (with 12 instead of 14 actin-binding sites) and to markedly different amino acid sequences at its $\mathrm{NH}_{2}$ and $\mathrm{COOH}$-terminal ends. Recent studies in our laboratory have demonstrated that troponin- $\mathrm{T}$ binds at two sites on skeletal TM. One of these sites (site 1) is close to or at the COOH-terminal end of TM and is $\mathrm{Ca}_{2}+$-insensitive. The other site (site2) is in a region about $1 / 3$ of the distance from the $\mathrm{COOH}$-terminal end and is sensitive to $\left[\mathrm{Ca}^{2+}\right]$ in the presence of troponin-C and -I. In the case of platelet TM, interaction and amino acid sequence studies have shown that site 1 is absent while site 2 is essentially identical to skeletal TM. Preliminary evidence suggests that a similar situation exists with smooth muscle TM even though the head-totail aggregation properties are even more pronounced than for skeletal TM. Supported by MRC of Canada.

\section{Calcium-Dependent Neutral Proteinase in Hamster Cardiac} and Skeletal Muscle.

JOHN S. ELCE and RENATE BARTH. Department of Biochemistry, Queen's University, Kingston, Ontario.

A $\mathrm{Ca}^{2+}$-dependent proteinase is present in many mammalian tissues, and has been implicated in turnover of muscle protein and in dystrophy, although this is not yet certain. In order to determine the probable substrates of the proteinase, its localization has been determined by the indirect immunofluorescence technique. The enzyme was purified from hamster carcass, and antibodies were raised against it in rabbits. The antisera inhibited the activity of the proteinase and appeared to be monospecific on rocket immunoelectrophoresis. More stringent tests of monospecificity are in progress, with [ $\left.{ }^{125} \mathrm{I}\right]$-labelled proteinase or antibody and the use of 2-D gel systems for maximum resolution. In frozen sections of hamster cardiac and skeletal muscle, fixed in $2 \%$ formalin, the proteinase was found to be located either at the plasma membranes of muscle fibres, or in the connective tissue (endomysium) between the fibres, but these alternatives could not be distinguished at the level of the light microscope. Extension of this work to the dystrophic hamster, and to the electron microscope with the use of immunoperoxidase and protein A-gold techniques, is under way. Supported by the M.D.A.C. and the Deutsche Forschungsgemeinschaft.

\section{Two Calcium Currents in Egg Cells.}

\section{A.P. FOX. Dept. of Physiology, UCLA, CA, USA.}

Two distinct types of voltage-activated calcium channels have been found in the unfertilized egg of the marine polychaete Neanthes arenaceodentata under voltage clamp-conditions. The currents through both channels relax with time under maintained depolarization; for one channel, $\mathrm{Ca}(\mathrm{I})$, the relaxation is due to a voltage-dependent inactivation while for the other channel, $\mathrm{Ca}$ (II) the relaxation is current-dependent. The relaxation for $\mathrm{Ca}(\mathrm{I})$ current follows a single exponential decay with time constants in the range of 10 to $50 \mathrm{~ms}$. $\mathrm{Ca}$ (II) current decay was found to be a function of $1 / \mathrm{t}$, with half-times of decay in the $200-600 \mathrm{~ms}$ range. The current-dependent relaxation appears to be due to depletion of calcium near the channel, much like the mechanism found for calcium currents in frog skeletal muscle. In both channels strontium and barium can replace calcium as the permeant divalent ion, but the permeability ratios as determined from peak inward currents are different for the two channels. Cobalt was found to block in both channels but was more effective on $\mathrm{Ca}$ (II). Cad- 
mium blocks $\mathrm{Ca}(\mathrm{II})$ but doesn't affect $\mathrm{Ca}(\mathrm{I})$. This evidence for the existence of two distinct types of calcium conductance in the same preparation may be of importance in resolving conflicting observations on calcium conductances in different preparations. Supported by MDAC pre-doctoral fellowship \#C790501.

\section{Phenotypic Alterations of Tonic Muscles Innervated by Nerves of Twitch Muscles in Dystrophic Chickens.}

A.C. GANDY, E. COSMOS, and J. BUTLER, McMaster University, Health Sciences Center, Hamilton, Ontario.

An early phenotypic expression of avian HMD is the inability of fasttwitch glycolytic (FTG) muscles to differentiate from immature to mature, enzymatic profiles during ex ovo development. Since slow-tonic (ST) muscles are spared signs of the disease, this implies that the disease expression is target specific. Therefore, we asked the question: If a genotypically dystrophic (D) ST muscle is asked to differentiate the mature enzymatic profile of a FTG muscle, will it express dystrophic phenotypes? Operations were performed on newly-hatched normal $(N)(n=20)$ and dystrophic $(n=20)$ chicks. With its origin intact, the ST anterior latissimus dorsi (ALD) was transposed to the contralateral side, sutured in two places to the triceps brachii, and coupled to the 'fast' superior brachialis nerve. Physiological, structural and histoenzymic analyses done from 2 to 12 weeks post-operatively indicate that the degree of conversion is variable with the greatest conversion, thus far, observed at 2 weeks in a N-ALD $(59 \%)$ and at 12 weeks in a D-ALD (73\%). Of greater interest is the observation that only cross-reinnervated ALD muscles of dystrophic genotype display dystrophic phenotypes. Supported by grants from MDAC and MDA.

The Relation between Axon, Myelin and Conduction Velocity during Atrophy of Mammalian Peripheral Nerves.

J. GILLESPIE, R.B. STEIN \& T. MILNER, Department of Occupational Therapy and Physiology, University of Alberta, Edmonton, Alberta.

The course of atrophy of cat hind-limb cutaneous and muscle nerves was followed for 273 days after the nerves were cut and ligated. Conduction velocity spectra, determined by the method of Milner and Stein (J. Neurol. Neurosurg. Psychiat, 1981, 44:485), were correlated with fibre size spectra, determined by light and electron microscopy. As atrophy progresses, conduction velocities decrease to a greater extent than do fibre diameters (axon and myelin), and correlate better with reduction in diameter of the axons. There is a linear relationship between axon diameter and total fibre diameter with decreasing slope over time. The ratio of axon and fibre perimeters remains constant and near the optimum value of 0.6 . During atrophy the axon shrinks and the myelin tube remains intact, flattening or infolding into the axon space without appreciably changing the inner perimeter, outer perimeter or thickness of the myelin. Changes in conduction velocity agree with the predictions of the Frankenhaeuser-Huxley equations (J. Physiol., 1964, 171:302), assuming this mode of atrophy. This mode of atrophy also provides optimum conditions for precise and complete regeneration of nerve fibres to muscles or sense organs. Supported by grants from MRC and MDAC.

Molecular Forms and Cytochemical Distribution of Acetylcholinesterase (AChE) in Skeletal Muscles of the 129/ReJ Dystrophic Mouse.

V. Gisiger, and H. Stephens, Department of Anatomy, Université de Montréal.

Our aim was to analyse changes in AChE in skeletal muscles of normal and dystrophic $(129 / \mathrm{ReJ})$ mice. We approached this problem by a concomitant cytochemical and biochemical study of consecutive transverse slices (1 mm in thickness) of contralateral muscles of the same animal. In the normal mouse the motor endplates are clustered together in a pattern whose three-dimensional configuration is highly reproducible and specific for a given muscle. In contrast, motor endplates are dispersed in dystrophic muscles and no clear pattern can be discerned. Similarly, there is a specific profile of molecular $\mathrm{AChE}$ forms for individual normal muscles, whereas the patterns resemble each other in dystrophic animals. The profiles of the dystrophic muscles are characterized by a marked reduction in the $G_{4}$ (10S) molecular form. The $A_{12}(16 S)$ form is also decreased to some extent, but its loss is compensated, at least in part, by an increase in the $A_{8}$ form. Another point that has arisen from our concomitant cytochemical and biochemical studies is that, for a given section, there is a strict correlation between the presence therein of motor endplates and the $A_{12}$ molecular form. This would suggest that, in the mouse, the $A_{12}$ molecular form is restricted to motor endplate regions, as has been described for numerous species. Supported by the MDAC and MRC. HRS is a scholar of the CRSQ.

\section{Lectins as Probes for the Study of Cell Surface Glycopro- teins of Normal and Dystrophic Fibroblasts.}

B.B.GORDON, C. GUERIN and S.D.J. PENA, Department of Neurology and Neurosurgery, McGill University and Montreal Neurological Institute, Montreal, P.Q.

A new methodology was developed to study the cell surface glycoproteins of cultured cells. This was based on the binding of a variety of biotinyl-lectins to nitrocellulose electro-blots of total cell proteins after separation in SDS-polyacrylamide gels, followed by visualization with avidin-biotinyl-peroxidase complexes. The technique proved to be very sensitive and a large number of glycoproteins were "stained" with concanavalin A (Con A) and wheat germ agglutinin (WGA). Binding of peanut agglutinin (PNA) and to a lesser extent that of Ricinus communis agglutinin (RCA I) were found to be dependent on prior removal of sialic acid residues from the glycoproteins. Since by treatment of intact viable cells with neuraminidase, only external sialic acid residues were removed, PNA and RCA I could thus be utilized for selective visualization of cell surface glycoproteins. Also, because PNA was known to bind preferentially to oligosaccharides with 0 -glycosidic linkages and RCA I to those with $\mathrm{N}$-glycosidic linkages, the two lectins were complementary.

This technique is currently being applied to fibroblasts of patients with Duchenne muscular dystrophy, myotonic dystrophy and other neuromuscular diseases and the results of these investigations will be reported. Supported by the MDAC and MRC of Canada.

\section{Temperature Dependence of Contractile Properties in Nor- mal and Dystrophic Mouse Muscles.}

T.GORDON and R.B. STEIN, Departments of Pharmacology and Physiology, University of Alberta, Edmonton, Alberta.

Isolated extensor digitorum longus (EDL) and soleus muscles from normal and genetically dystrophic (129/REJ) mice were studied at temperatures from about 8 to $38^{\circ} \mathrm{C}$. The rate constant for the exponential decay of tension during an isometric twitch or short tetanus was significantly reduced in dystrophic EDL muscles. However, the temperature dependence, measured from the Q10's and apparent enthalpies of activation for several parameters of isometric twitches and tetani (See Stein et al., this meeting) were similar in all muscles studied, although the values tended to be slightly higher in the slower soleus muscles than in the fast EDL muscles and in the dystrophic than in the normal muscles (1-2 $\mathrm{kcal} / \mathrm{mol}$ ). The reasons for the small differences are not known, but the same rate-limiting processes probably apply to fast and slow normal and dystrophic muscles.

One stimulus can facilitate the contractile response to a second or third stimulus. This facilitation (and the tetanus/twitch ratio) tended to be greater in dystrophic than in normal muscles at $20^{\circ} \mathrm{C}$, although facilitation (and the tetanus/twitch ratio) increased with temperature at about the same rate in all muscles. The implications of these results for theories of muscular dystrophy will be discussed. Supported by a grant from MDAC. 


\section{Conversion of Fast to Slow Fibers Mediated by Active Mus- cle Tension in Developing Lobsters.}

C.K. GOVIND, Scarborough College, University of Toronto, West Hill, Ontario.

In juvenile lobsters (Homarus americanus) the paired claw closer muscles are symmetrical consisting of a central band of fast fibers sandwiched by slow and intermediate fibers. During subsequent development one of the paired muscles converts its fast fibers to slow to become a crusher while the other muscle converts most of its slow fibers to fast to become a cutter. Which of the paired muscles becomes a crusher is determined in a random manner in the juvenile 4th and 5th stages. Rearing these juvenile stages with a variety of substrate which can be manipulated by the claws results in the formation of dimorphic claws. Rearing without a substrate suppresses formation of a crusher claw and of slow fibers and instead produces paired cutter claws. Immobilization or dactylotomy of the claw does not inhibit formation of a crusher claw whereas tenotomy of the closer muscle, with the resultant loss of passive (resting) and active muscle tension, does inhibit development of slow fibers. Treatments which allow the closer muscle to maintain its passive tension but reduce or eliminate active tension such as denervation, deafferentation and tenotomy of the antagonistic opener muscle also prevent formation of a crusher claw. These results suggest a nerve impulse-mediated influence in the conversion of fast fibers to slow during development. Supported by MDAC.

\section{Quantitative Analysis of Intramembrane Particle Distribu- tion in Plasma Membranes of Dystrophic Hamster Car- diomyopathy.}

K.A. GRAHAM, R.R. SHIVERS and B.G. ATKINSON. Cell Science Laboratories, Dept. of Zoology, Univ. of Western Ontario, London, Ontario.

The intramembrane particle (IMP) profile of both control and dystrophic (Syrian; Bio. 14.6) hamster cardiac muscle cell plasma membranes was assessed by freeze-fracture in order to determine whether this animal model for muscular dystrophy possesses the same structural characteristics as other more thoroughly studied models. Samples of cardiac muscle tissue obtained from dystrophic hamsters ranging in age from 1-13 months were freeze-fractured and the resulting platinum replicas photographed at initial magnifications of 30,000 diameters and enlarged to a final magnification of 80,000 diameters. Intramembrane particle numbers were determined for all tissue samples by counting the particles in randomly selected areas of PF face surface. Control heart cell plasma membranes exhibited an average of 1700 particles $/ \mu \mathrm{m}^{2}$. The dystrophic cardiac cell plasma membranes con tained an average of 1248 particles/ $\mu \mathrm{m}^{2}$; a value which is $30 \%$ lower than that for controls. Particle frequency in dystrophic muscle plasma membranes was the same for all age groups sampled. The $30 \%$ depression in particle frequency in dystrophic hamster heart is consistent with values published for myofiber plasma membranes from human dystrophic skeletal muscle and skeletal muscle from other animal models of the diseases, and supports the use of dystrophic hamster cardiomyopathy as a model for muscular dystrophy. In addition, this study has shown for the first time that the depressed population of IMP's in the cardiac muscle plasma membrane is present throughout the life of the animal (1-13 months) and is seen in every cell sampled. Supported by a grant from the MDAC.

\section{Study of the Further Heterogeneity of the MM Isozyme of Creatine Kinase.}

BENJAMIN G. GUSLITS and HANS K. JACOBS, Univ. of Manitoba, Fac. of Medicine, Dept. of Biochemistry, Winnipeg, M anitoba, Canada.

The existence of 3 cytosolic isozymes of creatine kinase (MM, MB and $\mathrm{BB}$ ) is well established. For the MM isozyme of creatine kinase (MM CK) subspecies have been observed recently. The number of native subspecies and the changes induced in these subspecies by serum remains to be resolved. In the present study separation of the various subspecies of the MM CK was achieved by agarose or polyacrylamide gel isoelectric focusing. MM CK from human skeletal muscle was resolved into 21 active subspecies. R abbit and beef MM CK's were also shown to have patterns of 21 bands. These results are compatible with the presence of 6 different subunit forms in skeletal muscle which by random association into dimers could produce 21 different subspecies of creatine kinase. Comparison of focusing patterns from human muscle obtained at operation and at autopsy showed that the subspecies were not produced by post-mortem autolytic changes. Two-dimensional isoelectric focusing of human MM CK yielded a diagonal pattern, ruling out ampholyte-protein interactions as reason of heterogeneity. Heat inactivated serum stabilized certain CK subspecies and produced one additional anodally migrating MM CK form. Fresh serum had combined inhibitory and stabilizing effects on the MM CK subforms and allowed identification of 4 new anodally migrating subspecies not present in fresh muscle. If muscle disorders have MM CK subforms different from those of normal muscle, a powerful new diagnostic tool may have been found. Supported by a grant from MDAC.

\section{Human Ankle Stiffness Dynamics: Effect of Muscle Fatigue.}

I.W. HUNTER, P.L. WEISS and R.E. KEARNEY. Biomedical Engineering Unit, Faculty of Medicine, McGill University, Montreal.

In isolated muscle, stiffness is known to increase almost proportionally with increases in isometric force. We have demonstrated that the same relation holds in the neuromusclar system controlling the human ankle by decomposing the ankle position-torque (stiffness) transfer function into elastic, viscous and inertial terms. The elastic and viscous stiffness terms increase with increases in tonic torque and decrease with increases in position perturbation amplitude. The inertial term remains constant.

In this paper changes in the elastic and viscous stiffness terms during fatiguing contractions are determined. The relative roles of excitationcontraction coupling dynamics versus muscle mechanical dynamics in determining observed functional changes during muscle fatigue are discussed. Supported by a Canadian Medical Research Council grant. IWH is a Canadian Muscular Dystrophy Association Postdoctoral Fellow.

Changes in the Distribution of Five Proteins in Dystrophic Skeletal Muscle: Identification of the Proteins, a Study of their Developmental Significance and Nerve-Dependence in Normal Animals.

L.G. JASCH, Dept. Anatomy, University of British Columbia, V ancouver.

Previous studies from this laboratory showed that during the progression of muscular dystrophy in the $\mathrm{C} 57 \mathrm{BL} / 6 \mathrm{~J} \mathrm{dy} / 21 / \mathrm{dy}^{21}$ mouse, the distributions of selected, but unidentified, proteins in the soleus and extensor digitorum longus (EDL) became highly altered. Recent studies, including twodimensional electrophoresis of proteins from these muscles, allow us to suggest that in the dystrophic soleus, the weight/weight ratios of myosin light chain proteins $\mathrm{LC} 1 \mathrm{~s} / \mathrm{LC} 2 \mathrm{f}$ and $\mathrm{LC} 2 \mathrm{~s} / \mathrm{LC} 2 \mathrm{f}$ are decreased compared to normal. In the dystrophic EDL, there is a decrease in the weight/weight ratios of the calcium binding protein parvalbumin/LC2 $\mathrm{f}$ and the phosphorylated form of LC2f/LC2f compared to normal. Between 1 and 4 weeks of postnatal development in normal mice, these ratios increase dramatically, apparently due to the increased accumulation of $\mathrm{LCls}$, LC2s, parvalbumin and LC2f-phos in the muscles. Our studies, and studies from other laboratories, suggest that this selective postnatal accumulation of proteins in the normal soleus and EDL is a nerve-dependent phenomenon. It is suggested that the changes in distributions of muscle proteins observed in dystrophic muscles could result from the failure of a necessary nerve-muscle interaction during postnatal development. Supported by MDAC.

Effect of Two New Thiol Protease Inhibitors on the Development of Heart Necrotic Changes in Polymyopathic Hamsters.

G. JASMIN and L. PROSCHEK, Département de pathologie, Faculté de médecine, Université de Montréal, Montréal.

There is now a substantial body of evidence that latent proteases are involved in the degradative pathway of myofibrillar proteins. Interestingly calcium-activated neutral protease (CANP) activity was found significantly increased in dystrophic muscles. We have investigated the effects of two 
newly synthesized thiol protease inhibitors, Ep475 and Ep459, on the progression of heart and skeletal muscle lesions in polymyopathic hamsters UM-X7.1. The findings demonstrate that these thiol protease inhibitors administered in vivo by means of osmotic minipumps can significantly reduce the development of heart necrotic changes. The myocardial $\mathrm{Ca}^{++}$content was found equally diminished after treatment with good preservation of sarcomeric architecture. The severity of skeletal muscle changes under the present experimental conditions was found unchanged. It may be inferred that CANP(s) are involved in the development of early myolytic heart changes and that these inhibitors offer interest for investigating muscle wasting diseases. Supported by a grant from MDAC.

\section{In Vitro Study of Growth and Differentiation of Normal and Dystrophic Human Skeletal Muscle.}

G. JASMIN, C. TAUTU and L. PROSCHEK, Département de pathologie, Faculté de médecine, Université de Montréal, Montréal.

Normal and dystrophic (Duchenne) muscle cultures were established by means of a proper explant technique. The basic evolutional stages of myogenesis were studied comparatively. As a rule, normal and dystrophic muscle fibers, once migrated from explants, multiplied and fused with formation of multinucleated myotubes and obvious maturation. There were however some distinctive features which summarize as follows: a) an earlier cell migration from explants; b) a weak or retarded and in some cases a complete absence of fusion; c) many atypical myogenic cells - as compared to conventional myoblasts - involved in the fusion process; d) an absence of spontaneous functional activity and a shorter longevity of the myofibers. These findings emphasize a deficient myogenesis and the extreme fragility of dystrophic muscle cells. Supported by a grant from MDAC.

Chronic Electrical Stimulation Fails to Induce Necrosis in Hind Leg Muscles of Cordotomized Dystrophic Hamsters (UM-X 7.1).

G. KARPATI, S. CARPENTER and ST, PRESCOTT, Montreal Neurological Institute - McGill University, Montreal, P.Q.

In hind leg muscles of UM-X 7.1 dystrophic hamsters, cordotomized in the prenecrotic stage (at age 17-18 days), no necrosis and little central nucleation of muscle fibers was present at age 45 and 60 days.

Hind leg muscles of 30-day-old cordotomized animals were stimulated under general anaesthesia for 3-8 days (about 8 hours a day) either directly (anterior tibial group) or through the exposed sciatic nerve. 1 second trains of supramaximal stimuli at $30-50 \mathrm{~Hz}$ were delivered at 2 second intervals by constant current external stimulator. Stimulation was suspended for 5 minutes at 15 minute intervals to avoid inexcitability of muscles due to fatigue. Despite this, no necrosis and little central nucleation of muscle fibers was present in the stimulated muscles (anterior tibialis, gastrocnemius, plantaris and soleus). These results suggest that electrical or mechanical activity of muscle fibers is only effective before 20 days of age in inducing the molecular basis necessary for the microscopic pathological expression of the dystrophic gene. Supported by a grant from MDAC.

\section{Regulation of Glucose Transport in L6 Muscle Cells in Culture.}

A. KLIP, W.J. LOGAN and G. LI. Dept. Neurology, Research Institute, The Hospital for Sick Children, Toronto.

Glucose transport in muscle is regulated by a variety of hormones, and by metabolic conditions inherent to this tissue. Moreover, glucose transport is deficient in certain muscular malfunctions. The structure of muscle tissue poses problems of accessibility and heterogeneity, which complicate the determination of the molecular mechanisms underlying regulation of transport. We have shown that the myoblast $\mathrm{L} 6$ cell line is a suitable model system of muscle, and we have characterized kinetically and pharmacologically the uptake of 2-deoxyglucose into these cells. The number of glucose carriers on the cell surface was determined by binding of the specific inhibitor Cytochalasin B.
In monolayer cultures, the transport rate of hexose decreased when cells approached confluency. The decrease in transport rate was reflected both in an increase in $\mathrm{Km}$ and a decrease in Vmax. Quantification of the number of Cytochalasin B-binding sites determined the change in V max is not due to a decrease in the number of glucose carriers. Cells grown in monolayers at different cell densities have been detached by trypsin; differences in the rate of hexose uptake (at $0.5 \mathrm{mM}$ ) persisted in suspension, suggesting that the effect of density on the transport system is permanent. Supported by a grant from MDAC.

\section{Studies of Duchenne Muscular Dystrophy (DMD) at the} Nucleic Acid Level.

C. KREIS, J.L. HAMERTON and K. WROGEMANN. Departments of Biochemistry and Pediatrics (Genetics) University of Manitoba, Winnipeg.

The discovery of restriction enzymes has provided us with the potential to discover new genetic markers throughout the human genome. Restriction enzymes recognize certain base sequences in DNA. Variants of these sequences will result in the disappearance of restriction enzyme cleavage sites or in the generation of new ones. With appropriate probes these can be recognized as "restriction fragment length polymorphisms" (RFLPs). In collaboration with Dr. B. White (Queen's University) we have begun to prepare DNA probes which are specific for unique sequences of the human X-chromosome (Gusella et al. Proc. Natl. Acad. Sci. 77, 2829 (1980)). Potentially the best probes map around band p-21 on the short arm of the $\mathrm{X}$-chromosome, the putative site of the DMD gene. Using these probes we are searching in large families with DMD for RFLPs which are linked to the DMD gene. Appropriate RFLP linkages will allow carrier detection and prenatal diagnosis of selected cases. If, on the other hand, the mutant protein for DMD has already been discovered (Wrogemann et al., this conference) it is possible to prepare a DNA probe for the DMD gene itself. Supported by the MDAC, MRC and Manitoba Health Research Council.

\section{Acetylcholinesterace (AChE) Molecular Forms in Murine Muscular Dystrophy.}

Michael H. Lindenbaum and Bruce G. Livett, The Montreal General Hospital and McGill University, Montreal, Quebec.

Alterations in AChE molecular forms have been demonstrated in affected muscles of 129ReJ dy/dy mice. Sung (1978a,b) found a decrease in the levels of the $16 \mathrm{~S}$ form and an increase in the $5 \mathrm{~S}$ form in whole hindlimb preparations. Skau et al (1980) found a similar decrease in the levels of $16 \mathrm{~S}$ $\mathrm{AChE}$ and as well, the absence of the $10 \mathrm{~S}$ form in extensor digitorum longus (EDL) muscles. We have extended these investigations to the $d y_{2}$ mutant and have compared the AChE molecular forms of both soleus and EDL muscles from 12 week old C 57BL/6J $\left(d y^{2 /} / d y^{2 J}\right)$ and $( \pm / \pm)$ mice. In the dystrophic EDL, the $10 \mathrm{~S}$ and $16 \mathrm{~S}$ forms were significantly decreased while in contrast, the $5 \mathrm{~S}$ form was significantly increased. Although demonstrating a higher total AChE activity the dystrophic soleus did not show significant changes in the $\mathrm{AChE}$ molecular forms. These changes indicate that a biochemical abnormality exists in the biosynthesis, assembly and/or maintenance of particular AChE molecular forms in the dystrophic EDL, and point further to a differential involvement of specific muscle groups in murine muscular dystrophy.

Skau, K.A. and Brimijoin (1980) Soc. Neurosci. Abstr. 6, 404.

Sung, S.C. (1978a) Life Sci. 23, 69-74.

Sung, S.C. (1978b) Muscle and Nerve 1, 157-161.

Supported by a grant from MDAC.

\section{The Influence of Calcium and Other IONs on Muscle Amino Acid Transport.}

By W.J. LOGAN, A. KLIP and E. GAGALANG, Division of Neurology, Department of Paediatrics, University of Toronto and the Hospital for Sick Children, Toronto, Ontario.

Muscle integrity is dependent on a specific ionic microenvironment. Small variations in ion concentrations can alter function. Certain amino acid transport systems are sensitive to ionic changes and may mediate 
some of the cellular dysfunction. This study was undertaken to determine the effects of ions on uptake of ${ }^{3} \mathrm{H}$-proline, (a substrate for transport system A) by L-6 muscle cells in culture.

Uptake of proline decreased about $50 \%$ when the extracellular medium was made $\mathrm{Ca}^{++}$-free. This decrease was not prevented by addition of $\mathrm{Mg}^{++}$at the same or higher concentrations. The dependence on extracellular $\mathrm{Ca}^{++}$was saturable in the physiological range of the cation's concentration. System A is $\mathrm{Na}^{+}$-dependent. Kinetic studies in monolayer cultures of $L-6$ cells reveal that V max is the parameter modulated by the availability of $\mathrm{Na}^{+}$. This suggests that the turnover of the transport site in the membrane is accelerated by $\mathrm{Na}^{+}$and that there are no changes in the affinity for proline since $\mathrm{Km}$ of transport remains unaffected. It is concluded that muscle amino acid transport systems can be influenced by ions. This may be an important regulatory mechanism in normal and/or diseased muscle. Supported by a grant from MDAC.

\section{Initiation of Translation in Control and Dystrophic Mice.}

D.M. NICHOLLS and K. NICKSON, Department of Biology, York University, 4700 Keele St., Downsview, Ontario M3J 1 P3.

Dystrophic mice of the C57B $1 \mathrm{dy}^{2 \mathrm{~J}} / \mathrm{dy}^{2 \mathrm{~J}}$ strain and of the ReJ $129 \mathrm{dy} / \mathrm{dy}$ strain and littermate controls were used to prepare initiation factors. The tissues were homogenized and a fraction was obtained which contained $40 \mathrm{~S}$ ribosomal subunits, as described by Sundkvist et al. (J. Biol Chem. $249,6512-6516,1974)$. The initiation factors were prepared from the $40 \mathrm{~S}$ subunits by a $0.5 \mathrm{M} \mathrm{KC1}$ salt-wash step and were assayed by the binding of ${ }^{35} \mathrm{~S} \mid$ methionyl-tRNA to control liver ribosomes. The eIF-2 activity was measured in brain, liver and muscle and in all of these tissues there was a significant decrease in the dystrophic mice. This decrease in initiation activity in muscle resembled the decrease in elongation activity of muscle cytosolic preparations previously reported by our laboratory. The results contrast with those we have found, e.g. in brain of rats exposed to lead toxicity or in liver or kidney undergoing hypertrophy. Supported by a grant from MDAC.

\section{Abnormal Distribution of Fibre Types in a Slow-Twitch Muscle of the Dystrophic Mouse During Postnatal Develop- ment.}

W.K. OVALLE, B.H. BRESSLER, L.G. JASCH, C.E. SLONECKER. Department of Anatomy, University of British Columbia, Vancouver, B.C.

The development of extrafusal fibres in the soleus muscle of $\mathrm{C} 57 \mathrm{BL} / 6 \mathrm{~J}$ $\mathrm{dy} 21 / \mathrm{dy}^{2 \mathrm{~J}}$ mice and their normal controls were studied by histochemical and quantitative methods at selected ages of $4,8,12$ and 32 weeks. While an adaptive ransformation of fibre types (FOG SO) was noted during normal postnatal growth, a striking alteration in total number and distribution of fibres characterized the dystrophic soleus. Between 4 and 32 weeks, a significant dimunition in overall fibre number occurred, and the absolute number and proportion of dystrophic SO fibres were drastically reduced. In contrast, the percentage of dystrophic FOG fibres increased steadily while their absolute numbers remained relatively constant. Atypical fibres in the dystrophic soleus were found in elevated numbers at all age groups, and probably reflect attempts at regeneration or an intermediate stage in fibre type conversion. Microscopically, both of the major fibre types appeared affected, albeit differently, by the dystrophic process. We suggest that a defect in the normal postnatal conversion and maturation of fibre types within the soleus occurs in this murine model for muscular dystrophy. Comparative studies on the companion fast-twitch EDL muscle are in progress. Supported by the MDAC.

\section{A Comparison of Fore-Limb and Hind-Limb Muscles in Dystrophic Mouse.}

\section{D.J. PARRY, Univ. Ottawa, Ottawa, Ont.}

Fast twitch muscles of the dystrophic mouse show prolonged times to peak tension (CT) and to half-relaxation $(1 / 2 \mathrm{RT})$ as well as reduced posttetanic potentiation (PTP). However, almost all work previously reported was carried out on hind-limb muscles which exhibit spontaneous twitching. It is well known that muscle properties may be changed by an altered ac- tivity pattern. I have, therefore, compared contractile, biochemical and histochemical properties of hind-limb and fore-limb muscles. These latter are clearly dystrophic but do not exhibit spontaneous twitching. The forelimb muscle ECRL is slowed but almost entirely due to a prolonged $1 / 2 \mathrm{RT}$ with relatively little change in CT. Both EDL and ECRL show decreased PTP. Fast twitch muscle of the hind limbs show the presence of slow myosin when immunohistochemistry is carried out using an antibody to slow myosin. No slow myosin is seen in ECRL. Surprisingly both EDL and SOL of dy2J mice contained less SDH than control muscles as did the fore-limb muscles ECRB and ECRL. Triceps brachii and the glycolytic portions of gastrocnemius showed increases, while the oxidative portions showed a decrease. Slow myosin may be synthesized in fast-twitch muscles of the hind-limb as a result of the altered activity pattern. Furthermore, doubt is cast on the suggestion that the spontaneous activity results in increased levels of oxidative enzymes. Supported by MDAC.

\section{Lectins as Cytochemical Probes for Visualization of Membrane Oligosaccharides in Human Muscle.}

S.D.J. PENA, B.B. GORDON, G. KARPATI and S. CARPENTER, Department of Neurology and Neurosurgery, McGill University and Montreal Neurological Institute, Montreal, Québec.

Biotinyl derivatives of seven plant lectins - concanavalin A (Con A), peanut agglutinin (PNA), Ricinus communis agglutinin (RCA I), Ulex europeus agglutinin I (UEA I), soybean agglutinin (SBA), Dolichos biflorus agglutinin (DBA), and wheat germ agglutinin (WGA), were bound to cryostat sections of biopsied normal human muscle and visualized with avidin-horseradish peroxidase conjugates. A distinct staining pattern was observed with each lectin which could be blocked by appropriate sugar inhibitors. The most general staining was observed with Con A, RCA I, and WGA, which permitted strong visualization of the plasmalemma-basement membrane unit, tubular profiles in the interior muscle fibers, blood vessels, and connective tissue. DBA was unique in providing good visualization of myonuclei. Neuraminidase pretreatment of the cryostat sections altered the pattern of staining by all lectins except UEA I and Con A in a manner which is in conformity with the known structure of the oligosaccharide chains of membrane glycoproteins and specificities of the lectins involved. Lectin histochemistry is a useful new tool for the investigation of muscle disorders. Supported by the MDAC and MRC of Canada.

\section{Application of Logistic Discrimination to Duchenne Muscular Dystrophy Carrier Detection.}

M.E. PERCY, M.W. THOMPSON, T. STUKEL* and D.F. ANDREWS*. Hospital for Sick Children and Department of Statistics*, University of Toronto.

In X-linked recessive disorders, heterozygotes (carriers) generally cannot be identified with $100 \%$ reliability by biochemical methods because of lyonization. In Duchenne muscular dystrophy (DMD), carrier detection is further complicated because the specific gene defect has not been identified. Individual non-specific tests such as serum creatine kinase identify only about $2 / 3$ of known DMD carriers. Our approach to improving the identification of DMD carriers has been to combine the results of more than one non-specific carrier test by means of logistic discrimination. This method is particularly suitable for separating populations of carrier and normal subjects on the basis of overlapping quantitative characteristics. The carrier probability assigned to a subject on the basis of her biochemical test results can be directly combined with her carrier probability from pedigree analysis to yield a final carrier risk. Other carrier tests can be readily substituted for, or used in addition to ones which have been used. Although our studies have improved the identification of carriers (Am. J. Med. Genet. 8: 397,1981; Am. J. Med. Genet., in press), very few subjects who may be normal can be classified as such with certainty. We are presently extending the technique of logistic discrimination in an attempt to identify non-carriers more accurately. Supported by MDAC, NSERC and the Research Institute, Hospital for Sick Children. 


\section{The Effect of Heat Shock on Gene Expression in Fibroblasts from Normal, Carrier and Dystrophic Mice and Hamsters.}

M. POLLOCK, B.B. ATKINSON and R.R. SHIVERS. Cell Science Laboratories, Dept. of Zoology, University of Western Ontario, London, Ontario.

Cultured fibroblasts from mice and Syrian hamsters exhibit a change in gene expression in response to a brief heat shock treatment. Both rodent cell types show an enhanced synthesis of polypeptides with Mrs of 64,000 and pl's of 5.4 and 5.8, and apparent de novo synthesis of a triad of polypeptides with $\mathrm{M}_{\mathrm{rs}}$ of 25,000 and $\mathrm{pl}$ 's of $6.2,5.8$, and 5.4. No differences are seen in the heat shock response elicited by fibroblasts from dystrophic (ReJ/129 mice and B10 14.6 hamsters), carrier and control animals. In each case, the heat shock induced polypeptides are extractable from the fibroblasts by Triton $X-100$. The similarity in response to heat shock between these two rodent species suggests a common mechanism for dealing with environmental stress; our data further suggest that qualitative differences in such 'stress management' do not contribute to the dystrophic condition. Supported by a grant from MDAC.

\section{Cytoskeletal Elements in Normal and Dystrophic Hamster Fibroblasts.}

M. POLLOCK, R.R. SHIVERS and B.G. ATKINSON. Cell Science Laboratories, Dept. of Zoology, University of Western Ontario, London, Ontario.

Muscle-derived fibroblasts from control and dystrophic (B10 14.6) Syrian hamsters were examined by indirect immunofluorescence using antibodies to tropomyosin, actin, $\alpha$-actinin and $\mathbf{M}$-line protein. Cells were observed at various times after plating. Although the distribution of cytoskeletal proteins varies as the cells attach and spread, no differences were detected between cells from control and dystrophic animals. Cytoskeletal elements in Triton X-100 extracted cells were also visualized directly using the method of Pena; again, no anomalies were seen in the dystrophic cells. These data are in agreement with recent work showing normal microfilament arrays in fibroblasts from dystrophic chickens and Duchenne dystrophy patients. Supported by a grant from MDAC.

\section{Structural Comparison of Two Forms of Rabbit Muscle Calsequestrin.}

R.A.F. REITHMEIER and B. COZENS, Department of Biochemistry, Univ. of Alberta, Edmonton, Alberta.

Calsequestrin, a calcium-binding protein present in the terminal cisternae of muscle sarcoplasmic reticulum, is thought to play an essential role in calcium storage and release. In order to fully understand the role of calsequestrin in the regulation of calcium levels in muscle cells at the molecular level, we have undertaken a completely structural study of this protein. Calsequestrin in rabbit muscle exists in two genetically determined forms, having different apparent molecular weights as determined by sodium dodecyl sulfate gel electrophoresis. Both forms have a similar amino acid composition. The amino-terminal sequence of Form $1(\mathrm{Mr}=44,000)$ was determined by automated Edman degradation as: $\mathrm{H}_{2} \mathrm{~N}$-Glu-Glu-Gly-LeuAsp-Phe-Pro-Glu-Tyr-Asp ${ }^{10}$-Gly-Val-Asn-Arg-Val-Ile-Asn-Val-Asn-Ala ${ }^{20}$ Ile-Asn-Tyr-Tyr-Lys-Asn-Val-Phe-Lys-Tyr ${ }^{30}$-Gly-Val-Leu-Ala-Leu-LeuTyr-Glu-Pro ${ }^{40}$ - Gly-Asp - Lys-Ala-. Form 2, which has a lower ap parent molecular weight, has an identical amino-terminal sequence. Forms 1 and 2 gave similar peptides by cyanogen bromide cleavage or tryptic cleavage of succinylated protein as determined by gel filtration. The two forms of calsequestrin therefore appear to have a very similar amino acid sequence. Further sequence analyses will lead to an elucidation of the structural difference between the two forms of rabbit muscle calsequestrin and will provide insight into the function of this protein in muscle contraction. Supported by MDAC.

\section{Two Functional Nicotinic Receptors of Different Affinity on Human Peripheral Blood Lymphocytes.}

MAREK ROLA-PLESZCZYNSKI, LOUIS MENARD and SIMON LEMAIRE, Immunology Division, Department of Pediatrics and Department of Pharmacology, Faculté de Médecine, Université de Sherbrooke, Sherbrooke, Québec.

To further understand the basic immunoregulatory defect present in myasthenia gravis, we studied nicotinic acetylcholine receptors (AchR) on human peripheral blood lymphocytes. Binding studies using ${ }^{125} \mathrm{I}$ Bungarotoxin suggested the presence of such AchR on at least some blood lymphocytes. Functional studies revealed the presence of possibly two distinct receptors. Thus we found a biphasic suppressive effect of nicotine on lymphocyte proliferative responses to $\mathrm{PHA}$, with peaks at $10^{-9}$ and $10^{-5} \mathrm{M}$. Conversely, a biphasic enhancing effect was observed with equimolar concentrations of d-tubocararine. When lymphocytes were fractionated on a nylon wool column, non-adherent cells (predominantly $\mathrm{T}$ lymphocytes) were only sensitive to $10^{-9} \mathrm{M}$ concentrations of nicotine or tubocararine, $10^{-5} \mathrm{M}$ producing little or no effect.

These studies suggest that distinct lymphocyte populations may bear high or low affinity AchR, with possible relevance to the pathogenesis of myasthenia gravis. Supported by a grant from MDAC.

\section{Dystrophic Murine Skeletal Muscle Z-Discs: High Voltage Electron Microscopy.}

R.R. SHIVERS, D.I. RODENHISER and B.G. ATKINSON. Cell Science Laboratories, Department of Zoology, University of Western Ontario, London, Ontario.

Attempts to correlate biochemical evidence for defective skeletal muscle proteins with the 3-dimensional arrangement of these proteins in sarcomere Z-discs of dystrophic mouse myofibrils have been frustrated by the extreme structural complexity of the tissue. High voltage electron microscopy therefore, has been employed to examine sarcomere $\mathrm{Z}$-discs in $1 / 8$ micron plastic sections of both isolated myofibril fragments and whole tissue in order to visualize the spatial arrangement of $\alpha$-actinin, tropomyosin and actin in the Z-disc. Biochemical evidence suggests that one of these structural proteins, $\alpha$-actinin, is quantitatively and/or qualitatively altered in muscular dystrophy and consequently, may contribute to sufficient structural instability in the supramolecular architecture of Z-discs to account for the disorganization of the disc (streaming) and sarcomere characteristic of muscular dystrophy. Stereo electron micrographs of non-dystrophic mouse muscle Z-discs generally support the notion that actin filaments end within the disc and are anchored in the disc by "cross-bridges" (tropomyosin?) and by molecules of $\alpha$-actinin. This arrangement constitutes the paracrystalline lattice-work of the discs that is seen in cross-sections of myofibrils. Our current high voltage electron microscope studies employ immunochemical methods with gold labelling and are specifically designed to identify $\alpha$-actinin and tropomyosin in the $\mathrm{Z}$-dics of both normal and dystrophic mouse skeletal muscle. Supported by a grant from the MDAC.

\section{Segmental Myofibre Necrosis in Myotonic Dystrophy.}

MEREDITH M. SILVER, DIPONKAR BANERJEE and ARTHUR J. HUDSON. Department of Pathology, St. Joseph's Hospital, London, Ontario.

By immunohistochemical staining methods, we identified immunoglobulins in muscle cells from stored tissue derived from either muscle biopsies or autopsies on patients with myotonic as well as other forms of muscular dystrophy, and on patients with a variety of non dystrophic muscle diseases. We localized, by light microscopic morphology, the immunoglobulin staining to areas of necrosis within myofibres in 9 of 19 dystrophic and 11 of 27 non dystrophic subjects. We believe that the staining represented a nonspecific diffusion process. Our study helped us distinguish artefactual contraction bands from segmental myofibre necrosis and the latter from coagulation necrosis. Segmental myofibre necrosis was seen frequently but coagulation necrosis was absent in myotonic dystrophy. Supported by Grant No. M335Al from the Muscular Dystrophy Association of Canada. 


\section{Thymic Alterations in Genetically Dystrophic Mice.}

C.E. SLONECKER, W.K. OVALLE, B.H. BRESSLER, L.G. JASCH. Department of Anatomy, University of British Columbia, Vancouver, B.C.

Whole thymic transplants from male C57BL/6J dy $2 / / \mathrm{dy}^{2 \mathrm{~J}}$ mice to neonatally thymectomized $\mathrm{C} 57 \mathrm{BL}$ recipients demonstrated marked histological changes after 8 weeks in situ. The thymic transplants were well vascularized but failed to reconstitute their lymphoid component of cells. The transplant contained a variety of acinar cells which demonstrated different capacities for secretion. The glands also contained large ductless, cyst-like follicles with abundant accumulations of lipid staining material. Ultrastructural examination of the secretory acinar cells showed that they contain large amounts of smooth endoplasmic reticulum and mitochondria. They resembled acinar cells that are typically associated with steroid synthesis. A comparison of glandular material within the mice revealed that these transplanted thymuses were histologically similar to the preputial gland. This gland is influenced by testosterone and is believed to produce steroids and pheremones. Thymic transplants from dystrophic female mice showed normal development in the host environment. These findings suggest that hormonal interactions with thymic tissue in dystrophic mice may influence the expression of dystrophic traits in mice. Supported by MDAC.

\section{What Limits the Rise and Fall of Mammalian Muscle Contractions?}

R.B. STEIN, T. GORDON and J. SHRIVER. Departments of Physiology, Pharmacology and Biochemistry, University of Alberta, Edmonton, Alberta.

The temperature dependence of contractions in isolated rat and mouse extensor digitorum longus (EDL) and soleus muscles was studied over the range of 8 to $38^{\circ} \mathrm{C}$. The values of: 1) the rate constant for the exponential rise of tension during an isometric tetanus, 2) the half-contraction time, 3) contraction time and 4) maximum rate of rise of tension during an isometric twitch and 5) the maximum shortening velocity in an isotonic contraction all had a $Q_{10}$ of about 2.5 (corresponding to an enthalpy of activation $\Delta \mathrm{H} \dagger=16 \mathrm{kcal} / \mathrm{mol}$. The $\mathrm{Mg}^{++} \mathrm{ATP}$ ase rates of myofibrils prepared from rat EDL and soleus muscles had a steeper temperature dependence $(\triangle H \dagger=31 \mathrm{kcal} / \mathrm{mol})$, but much lower absolute rates at $20^{\circ} \mathrm{C}$ than the rate of rise of tension. This suggests that the $\mathrm{Mg}^{++} \mathrm{ATPase}$ cycle rate is not limiting for force generation.

The rate constant for the exponential decay of tension during an isometric twitch or short tetanus (and the half-fall time of a twitch) had a break point at about $20^{\circ} \mathrm{C}$, with apparent values of $\triangle \mathrm{H} \dagger=28 \mathrm{kcal} / \mathrm{mol}$ below $20^{\circ} \mathrm{C}$ and $\triangle H \dagger=17 \mathrm{kcal} / \mathrm{mol}$ above $20^{\circ} \mathrm{C}$. The break point and the values of $\Delta \mathrm{H} \dagger$ at high and low temperatures agree closely with published values for the $\triangle \mathrm{H} \dagger$ of the sarcoplasmic reticulum (SR) $\mathrm{Ca}^{++}$ATPase. Thus, the fall of tension during a twitch or a short tetanus appears to depend directly on the rate of reabsorption of $\mathrm{Ca}^{++}$into the SR. Supported by a grant from MDAC.

\section{Ultrastructural Studies in Duchenne Muscular Dystrophy.}

\section{H. STEPHENS, Department of Anatomy, Université de Montréal.}

Previous light microscopic studies provided evidence that the terminal innervation patterns are modified in muscle from patients suffering from Duchenne muscular dystrophy (Acta Neuropath. 5: 225, 1965). In particular, the distribution of motor endplates, as revealed by acetylcholinesterase (AChE) activity, is abnormal. Our morphometric studies on cryostat sections of muscle biopsies from these patients indicate that there are more endplates per total muscle area and that the incidence of abnormal AChE activity is increased. AChE reaction product as well as collagen types III, IV and V can be detected within 'splits' of 'hypertrophied' fibers. (Nature 284: 470, 1980). Various degrees of 'splitting' can be seen ultrastructurally; the basement membrane is thickened in these areas, collagen fibers may be present and subsarcolemmal tubulovesicular structures are numerous. Regenerating muscle fibers are also surrounded by large amounts of collagen. Finally we are examining serially sectionned muscle (several hundred sections) from a Duchenne patient, from blocks that show a large percentage of intramuscular nerve fibers, in order to evaluate possible abnormalities in the terminal innervation pattern. Excessive axonal glycogen accumulation is present but this seems to be a non-specific finding. Supported by the MDAC. HRS is a scholar of the CRSQ.

\section{Carriers of Duchenne Muscular Dystrophy: Effects of Exer- cise.}

H. STEPHENS, L. REYNOLDS, M. VANASSE, J. PATERSON, G. HALL, S. BLOOM, K. WHITTAKER and V. DUBOWITZ, Jerry Lewis Muscle Research Centre, London, England.

The present study was undertaken to examine the effect of exercise on the metabolic response in carriers of Duchenne muscular dystrophy. Seven definite and 8 possible carriers of Duchenne muscular dystrophy (DMD) were studied. These females and their 9 age-matched controls performed a progressive power output test on a cycle ergometer to determine their maximal work capacity. A week later, they cycled for 15 minutes at one-half their maximum capacity, rested for 5 minutes and then re-attained their maximum. Blood samples were taken pre-exercise, at the 10th minute, before the second bout, immediately post-attainment of the maximum and 30 minutes and 24 hours post-exercise. During the exercise the inspired air volume and expired gas volume and the electrocardiograph were continuously monitored. From this information the cardiac frequency, ventilation, oxygen intake and carbon dioxide output and other ventilatory and cardiac parameters were calculated. Blood lactate levels rose 5 to 7 fold whereas glucose and free fatty acids exhibited only minor variations in both groups. There did not appear to be any difference in the minimal increase of creatine phosphokinase activity found in carriers and controls. No significant abnormality was found in the exercise response of the carriers, although their work capacity was slightly lowered. Supported by the MDAC and the MDG.

\section{Abnormal Immune Response in Murine Muscular Dystrophy.}

MYRON SZEWCZUK, Department of Microbiology \& Immunology, Queen's University.

The age-related changes in immune function in normal (nl) and dystrophic (dy) $\mathrm{C} 57 \mathrm{Bl} / 6 \mathrm{~J}$ mice were studied. Results show that 1 -mo-old dy mice produced a reduced antibody-forming cell (PFC) response to a thymic-dependent antigen. At 2-mo age, an enhance PFC response was observed as compared with nl controls. Older dy mice produced a diminution of immune reactivity. Using cell transfer procedures, we found that splenic B-cells from dy donors were $\mathrm{nl}$ in their capacity to produce an immune PFC. In contrast, the capacity of thymic cells from dy donors to facilitate helper T-cell function was abnormal. To substantiate this finding, dy mice in response to thymic-independent antigen produced an immune PFC, indistinguishable from $\mathrm{nl}$ controls. These findings support the hypothesis that an abnormal immune response of dy mice is influenced by a defective maturation of thymic function. Preliminary studies with dy mice have shown that there is a lack of auto-anti-idiotypic immunoregulation. This finding may reflect a defective thymic function since auto-antiidiotypic antibody production is thymic-dependent. The implications of this finding to muscular dystrophy may reflect a loss of regulatory control on effector cells, e.g. cytotoxic T-killer cells or perhaps natural killer cells which might recognize dy muscle as malignant and destroy them or prevent from dividing normally. Studies are in progress. Supported by MDAC.

The Application of Direct Tissue Isoelectric Focusing to the Study of Diagnostic Needle Muscle Biopsies.

B.J.THOMPSON, A.H.M.BURGHES, M.J. DUNN and V. DUBOWITZ. Jerry Lewis Muscle Research Centre, Department of Paediatrics and Neonatal Medicine, Hammersmith Hospital, London, England.

Experience since 1974 at the Jerry Lewis Muscle Research Centre has shown that needle muscle biopsies provide tissue of sufficient quantity and quality for routine diagnostic purposes. In addition, routine and freeze frac- 
ture electron microscopy can be performed successfully on a portion of each biopsy. When necessary, additional biopsy pieces (about $100 \mathrm{mg}$ of tissue) can be assayed for specific metabolites (e.g. carnitine). Moreover, besides the patients' ready acceptance of the technique, tissue from normal, healthy volunteers can be easily obtained.

However, the small amount of tissue available from clinical biopsy material in general and from needle muscle biopsies in particular, can place considerable limitations on the scope of the biochemical analyses that can be performed. In an effort to overcome some of these limitations we have developed a modified method of direct tissue isoelectric focusing in agarose which utilizes cryostat sections from diagnostic needle muscle biopsies. The method employs a broad $\mathrm{pH}$ gradient using a blend of commercially available ampholytes, with conditions modified to enhance anodic and cathodic resolution. Currently, a quantitative comparison of protein maps so obtained is underway. Also, the method has been demonstrated to be readily applicable to the study of isoenzymes of creatine kinase, glucose-6phosphate dehydrogenase, lactate dehydrogenase and hexokinase. Furthermore, a direct correlation with the histologic appearance is possible. Supported by a Postgraduate Research Fellowship from MDAC, an equipment grant from the Royal Society (U.K.) and by the Muscular Dystrophy Group of Great Britain.

\section{Effects of Ageing on Neuromuscular Function in Man.}

A.A. VANDERVOORT*, A.J. McCOMAS. McMaster University Medical Centre, Hamilton, Ontario.

Decreased strength during maximal voluntary contraction (MVC) has been reported as one of the consequences of ageing. The relative contributions to this decrease of incomplete neural activation (including subject motivation) and of muscle atrophy have been examined in healthy men and women aged 65-70 years. In comparison with younger adults, MVC torque was diminished in both plantar-flexor (PF) and dorsi-flexor (DF) muscles. However, completeness of neural activation, assessed by a twitch interpolation technique, did not appear less in the elderly. Isometric twitch recordings revealed a greater torque relative to MVC, and a longer contraction time, in elderly PF muscles; similar changes were not observed in the tibialis anterior. These preliminary results suggest that while excitable muscle mass and MVC decrease with ageing, other changes besides simple muscle atrophy occur in PF muscles. We wish to extend these studies by examining subjects in the 8 th and 9 th decades and by investigating muscle histology post-mortem.

\section{Contractile Properties of Denervated Skeletal Muscle.}

D.M.S. WEBSTER, B.H. BRESSLER. Department of Anatomy, University of British Columbia, Vancouver, B.C.

The effects of denervation on the isometric contractile properties of the fast-twitch EDL and the slow-twitch SOL muscles of the C57BL/6J +/+ mouse were investigated in vitro at $37^{\circ} \mathrm{C}$. Adult male animals were studied at $1,28,84$ and 210 days following unilateral section of the sciatic nerve. The maximum tetanus tension in abeolute terms, was reduced when compared to age matched controls at all time periods for the SOL and at 28 days and beyond for the EDL. Although twitch tension was less at most time periods, the denervated SOL exhibited a potentiated twitch tension at 28 days, and a potentiated twitch and tetanus tension at 28 days postdenervation when tension was expressed relative to dry muscle weight. The normalized tetanus tension for the denervated EDL was the same or greater than controls at all time periods. The normalized twitch tension of the denervated EDL was increased at 28 and 84 days. For both the denervated EDL and SOL, time-to-peak twitch tension and half-relaxation was significantly prolonged by 28 days and this continued to the older age groups. The fact that slow-twitch muscles may be more adversely affected by removal of their nerve supply than fast-twitch muscles will be discussed. Supported by MDAC and NAHS Grant, UBC.

\section{Progress in the Search for Mutant Protein(s) in Duchenne Muscular Dystrophy (DMD).}

K. WROGEMANN, E. ROSENMANN, C. KREIS, M. DOBBS and R.G. THOMPSON. Departments of Biochemistry and Pediatrics (Genetics) University of M anitoba, Winnipeg.

Based on two assumptions, namely that DMD is expressed in cultured skin fibroblasts and that the primary genetic defect manifests itself in (a) protein(s) which is (are) structurally altered or absent, we have been searching extensively for mutant protein(s) in DMD. Our most effective approach so far has been the use of 2-dimensional gel electrophoresis in combination with dual labeling techniques. In 8 patients with DMD we have found a "missing protein" of approx. 56,000 dalton and isoelectric point of approx. 6.7. Presently there is no single test that can establish the significance of this protein in the pathogenesis of DMD. However, if it were the primary defect it should be an X-linked protein, it should show Lyonization in fibroblasts from carriers of DMD and it probably should also be found in human skeletal muscle. Supported by the MDAC, Manitoba Heart Foundation and Winnipeg Clinic Research Institute. 